\title{
Stellate ganglion block for the treatment of intractable hiccups \\ - A case report -
}

Received September 14, 2017

Revised 1st, October 12, 2017

2nd, October 18, 2017

Accepted October 23, 2017

\section{Corresponding author}

Young Woo Cho, M.D., Ph.D.

Department of Anesthesiology and

Pain Medicine, Ulsan University

Hospital, 877 Bangeojinsunhwan-

doro, Dong-gu, Ulsan 44033, Korea

Tel: 82-52-250-8461

Fax: 82-52-250-7249

E-mail: ywcho67@gmail.com

\section{ORCID}

http://orcid.org/0000-0001-9683-1367

\section{Hee-won Son, Young Woo Cho, Young-ung Kim, and Yong-joon Shin}

Department of Anesthesiology and Pain Medicine, Ulsan University Hospital, Ulsan, Korea

\begin{abstract}
Hiccups are an involuntary contraction of the diaphragm that may repeat several times per minute. In general, hiccups are very common, transient, and self-limited. However, if the condition persists longer than days or months, it impacts a patient's quality of life. Pharmacologic and non-pharmacologic methods are used for the treatment of persistent or intractable hiccups. Nerve block and stimulation have been shown to be effective through neural pathway interruption or stimulation of the hiccup reflex arc. Stellate ganglion block (SGB) is an injection of local anesthetic adjacent to a group of nerves in the neck known as the stellate ganglion. The authors report a case of SGB as an effective treatment for a patient with intractable hiccups resulting from right lateral medullary syndrome.
\end{abstract}

Key Words: Hiccup, Lateral medullary syndrome, Stellate ganglion, Sympathetic nervous system.
A hiccup is the involuntary and sudden contraction of the diaphragm and intercostal muscles, followed by abrupt glottic closure, generating the characteristic sound. Hiccups are usually common, transient, and self-limited [1,2]. However, if the condition persists longer than days or months, it can negatively impact a patient's quality of life, possibly leading to dehydration, exhaustion, fatigue, malnutrition, insomnia, weight loss, depression, anxiety, and rarely death due to ventricular dysrhythmia $[3,4]$. Moreover, persistent or intractable hiccups make it difficult to maintain the body motionless, and thus interrupt radiologic imaging examinations such as magnetic resonance imaging (MRI) and positron emission tomography - computed tomography (PET-CT) scans.

A wide range of pharmacological and non-pharmacological treatments has been used for the management of persistent or intractable hiccups. Drugs commonly recommended for persistent or intractable hiccups have included chlorpromazine, metoclopramide, baclofen, gabapentin, carvedilol, 5-hydroxytryptamine (5-HT) agonists, olanzapine, midazolam, and amantadine $[1,2]$. In addition, in cases of intractable hiccups that are resistant to pharmacological therapy, various alternative non-pharmacological procedures have been applied. These procedures include phrenic or vagus nerve block, phrenic and/or vagus nerve stimulation, ultrasound-guided pulsed radiofrequency ablation of the phrenic nerve, acupuncture, and cervical epidural block [5-7]. Unfortunately, however, despite numerous attempts to develop treatments for persistent or intractable hiccups, including pharmacologic or non-pharmacologic methods, the optimal treatment has not been established because of the low incidence of hiccups, which makes case reports or randomized controlled trials relatively rare.

We experienced a case of remission of hiccups following repetitive stellate ganglion block (SGB) in a patient who was

This is an Open Access article distributed under the terms of the Creative Commons Attribution Non-Commercial License (http://creativecommons.org/licenses/by-nc/4.0) which permits unrestricted non-commercial use, distribution, and reproduction in any medium, provided the original work is properly cited.

Copyright (c) the Korean Society of Anesthesiologists, 2018 
unable to undergo MRI and PET-CT scans due to involuntary body movement induced by recurrent intractable hiccups despite continuous medication with baclofen and gabapentin.

To our knowledge, the case presented here is the first report of SGB as an effective treatment in a patient with intractable hiccups resulting from right lateral medullary syndrome (LMS).

\section{CASE REPORT}

A 66-year-old man presenting with suspected common bile duct (CBD) cancer was transferred to our hospital for further evaluation. Performance of MRI and PET-CT for further examination was impossible owing to the patient's continuous hiccups. Therefore, the patient was referred to the pain clinic for the treatment of the hiccups.

The patient had been diagnosed four years earlier with right LMS, also known as Wallenberg syndrome and had experienced intractable hiccups since that time. Initially, the hiccups were intermittently sustained for 3 to 4 days, but the duration of the hiccups became prolonged, and the patient was prescribed chlorpromazine $100 \mathrm{mg}$ daily. The hiccups then appeared to abate, but they subsequently recurred. About 2 years previously, in another hospital, while the patient was undergoing treatment with baclofen $10 \mathrm{mg}$ tid and gabapentin $300 \mathrm{mg}$ tid, continuous cervical epidural block using an epidural catheter was performed for about 10 days, and his hiccups were stopped. However, after 5 to 6 months, the patient's hiccups began to occur and stop repeatedly despite the continuous administration of baclofen and gabapentin, although he considered this pattern and frequency tolerable. His hiccups were then aggravated following an endoscopic retrograde cholangiopancreatography (ERCP) that was performed in an attempt to identify the cause of fever, general myalgia, and jaundice that had occurred a few days prior to his current presentation.

As the patient had a history of the cessation of hiccups following cervical epidural block, we considered performing a cervical epidural block. However, the risk of spinal cord injury due to the epidural needle was judged to be high owing to the motion of the patient's body caused by the continuous hiccups occurring more than 30 times per minute. Therefore, we decided to attempt SGB, as it is easier to maintain the patient's posture during this procedure, and it can be performed more simply and quickly. Because the patient's hiccups developed after the onset of the right LMS, we felt that SGB would be helpful. Therefore, we performed the SGB.

The patient was laid down in the supine position and a pillow was placed under the shoulder for cervical extension to facilitate palpation of the bony landmarks. After preparing the skin, the needle was inserted blindly using an anterior paratracheal approach technique and was advanced until it contacted the left transverse process of the C6 vertebra. At that time the needle was withdrawn slightly from the periosteum. After negative aspiration, $8 \mathrm{ml}$ of $0.2 \%$ ropivacaine was slowly injected for left SGB with repeated aspiration. Thereafter, the patient was observed for signs of Horner's syndrome to confirm successful SGB.

The patient was stabilized on the bed for 20 minutes following the procedure and returned to the ward after confirmation that no serious adverse events or complications had occurred. A few hours after the SGB, the hiccups stopped, but they recurred after about 12 hours. SGB was repeated on the left side the following day. As with the first SGB, the hiccups stopped after several hours. PET-CT was performed safely that same day, and there was no recurrence of hiccups until the next day. However, MRI was scheduled for confirmation of CBD cancer, so left-sided SGB was performed once more prophylactically, and the MRI was performed safely without recurrence of hiccups. The patient was diagnosed with CBD cancer and scheduled to undergo pylorus-preserving pancreatoduodenectomy (PPPD) 10 days later, and was discharged without hiccups. No hiccups were reported prior to the patient's admission to the hospital for PPPD, and the operation was performed the next day. However, the patient's hiccups occurred again from the third postoperative day, and stopped following the performance of SGB for four days, alternating the two sides once a day. No prominent side effects due to the procedure were observed.

Baclofen and gabapentin were tapered and discontinued after 3 postoperative weeks without any hiccups. The patient was discharged without further hiccups, with the exception of a sudden episode of weak hiccups that occurred for several hours and ceased spontaneously. In telephone interviews at 6 months and 10 months after discharge, the patient reported experiencing no further hiccup following discharge. 


\section{DISCUSSION}

Hiccups are usually transient and self-limiting. Potential causes of transient hiccups include distension and irritation of the stomach caused by overeating or carbonated drinks, spicy food, air swallowing, sudden changes in food temperature, alcohol, smoking, and psychogenic alterations such as excitement, anxiety, and stress. A case of hiccups lasting for more than 2 days is termed persistent, and a case of hiccups lasting for more than 2 months is termed intractable [2]. The presence of persistent or intractable hiccups is often associated with serious underlying organic pathology. Numerous causes of persistent or intractable hiccups have been well documented. Reported etiologies of these can be categorized as follows: lesions of the central nervous system (CNS); irritation of the peripheral nervous system (e.g., phrenic, vagal, and sympathetic nerves) induced by abdominal, thoracic, ear, nose, and throat disease; and metabolic, pharmacologic, surgical, infectious, and psychogenic factors $[1,2]$. According to a study by Keane [8], hiccups of CNS origin usually occur in men, with infarcts ( $70 \%$ of cases) the most common cause and the medulla (66\% of cases) the most frequent location. Hiccups occur in about $15 \%-25 \%$ of cases of LMS, which is the leading CNS cause of hiccups. In the present case, it is assumed that the patient experienced intermittent and repeated hiccups due to LMS, and the endoscopic irritation caused by ERCP further exacerbated the hiccups.

Although the pathophysiology of hiccups is not well defined, the hiccup reflex arc has been considered to play an important role. The hiccup reflex arc consists of the afferent limb, central hiccup center, and efferent limb. The afferent limb comprises the phrenic nerve, the vagus nerve, and the thoracic sympathetic chain arising from T6-T12. The areas involved in the central hiccup center include the proximal spinal cord (C3-C5), the brain stem respiratory center, the reticular activating system in the medulla oblongata, and the hypothalamus. Among the neurotransmitters involved in the process of hiccups, both dopamine (D) and gamma-aminobutyric acid (GABA) have been documented [9]. The efferent limb is composed of the phrenic nerve to the diaphragm (C3C5), innervation of the anterior scalene muscles (C5-C7), the accessory nerves to intercostal muscles (T1-T11), and the recurrent laryngeal branch of the vagus nerve to the glottis. Persistent or intractable hiccups generally occur through various abnormal processes affecting some components of the reflex $\operatorname{arc}[1,2]$.

Although the exact mechanism by which SGB terminates hiccups is not known, we would like to suggest some possible mechanisms that may underlie the function of SGB in the hiccup reflex arc.

First, we will explain how SGB can act on the afferent limb. One of the causes of hiccups is the excitement of the sympathetic nervous system $[1,2]$. In the course of ERCP, endoscopic irritation activates the sympathetic nervous system, which may lead to hiccups. Thus, there is a possibility that the hiccups stop with the blockade of the sympathetic nervous system by the SGB. Accurately speaking, SGB is a lower cervical sympathetic block or upper thoracic sympathetic block, in which a certain amount of local anesthetic is injected and diffuses into adjacent structures, resulting in cervico-thoracic denervation $[10,11]$. In the case of this patient, the sympathetic nerve block due to SGB may have been effective on the hiccup reflex arc. The sympathetic fibers at the thoracic level, originating from the spinal cord, enter the sympathetic chain to the brain through the stellate ganglion and it is possible that the SGB inhibited the hiccup reflex arc during this process [11-13].

Second, we will explain how SGB works on the central hiccup center. Lipov et al. [14] reported that SGB is effective in the treatment of hot flashes and posttraumatic stress disorder (PTSD) as well as complex regional pain syndrome (CRPS), and the authors proposed a mechanism of action of SGB based on the published evidence. The current hypothesis for this potential mechanism of action for SGB is as follows. The stellate ganglion has polysynaptic neural connections with various parts of the brain, such as the hypothalamus, amygdala, and insular cortex. The body responds to acute and chronic stress by increasing levels of nerve growth factor (NGF). Increases in intracerebral NGF lead to retrograde transport of NGF to the stellate ganglion. Moreover, increased NGF in the stellate ganglion promotes sprouting and nerve growth at the sympathetic end terminals, which, in turn, leads to increased brain norepinephrine (NE) levels triggering CRPS, hot flashes, and PTSD. Injection of local anesthetic to the stellate ganglion reverses this cascade. SGB induces the reduction of NGF, which leads to a reduction of sympathetic sprouting and eventually a decrease in NE levels. Thus, the effect of SGB is believed to contain not 
only neural inhibition in its sphere of innervation but also central and systemic sympathetic nervous system modulation. Acupuncture is considered to also modulate the hiccup reflex arc, although its exact mechanism remains unclear. Most acupoints for the treatment of hiccups are located near dermatomes related to the afferent or efferent pathways, secondary synapses, and nuclei involved in the hiccup reflex arc. Locally, acupuncture may interrupt the pathways of the reflex arc by changing blood perfusion, activation of the autonomic nervous system, or regulation of inflammatory mediators, or by altering axonal excitability. Interestingly, acupuncture can also influence the hiccup center at a distance by changing the secretion of neurotransmitters and neurohormones such as endogenous opioids, NE, serotonin, Substance P, and GABA $[2,6]$. Thus, an unknown mechanism of action, similar to the hypothetic mechanisms of SGB for PTSD and acupuncture for hiccups, is assumed to underlie the effectiveness of SGB in treating hiccups. Furthermore, NE only, or several neurotransmitters including NE, are speculated to be involved in the sympathetic nervous system modulation that can be mediated centrally in the brain. In other words, it is possible that in the present case, SGB neurologically modulated the brain to affect the neurotransmitters, and this caused the hiccups to stop. Following the first hypothesis, we speculate that the SGB may have aided in the performance of the MRI by stopping the hiccups within a short time, only a few hours. Following the second hypothesis, after the MRI, CBD cancer was diagnosed, and elective PPPD was performed 10 days later. On the third postoperative day, hiccups recurred, and bilateral SGBs were performed alternately every other day for 4 days. Thereafter, the patient was discharged without any additional hiccups, and no hiccups occurred for months. This result indicates that SGB has a potential long-term effect on hiccups. Lipov et al. [14] reported that SGB led to a prolonged reduction of NGF and eventually a decrease in NE, as well as prolonged efficacy of SGB for the treatment of CRPS, hot flashes, and PTSD. In addition, the hypothesis of this longterm effect provides a plausible explanation for the prolonged effect of local anesthetic markedly beyond the length of the half-life expected by the pharmacokinetics of local anesthetics. However, whether the sphere of action should necessarily include the hiccup center of the hiccup reflex arc in the brain will require further research.

Finally, we will explain how SGB works on the efferent limb in the hiccup reflex arc. Phrenic nerve blocks that constitute the efferent limb as a treatment for hiccups have been used occasionally. Because SGB was implemented with a blind technique in the present case, a rather large amount of local anesthetic was used, and this anesthetic may have spread around the injection site. It is possible that the 3rd to 5th cervical spinal nerves forming the phrenic nerve and the 5th to 7 th cervical spinal nerves innervating the anterior scalene muscle were blocked and stopped the hiccups. Unfortunately, we have been using SGB as a blind technique for a long period of time in general outpatient care, and have been using a somewhat larger amount of local anesthetic than the quantity used in ultrasound (US)-guided SGB. If US-guided SGB were performed, we would have used a small amount of local anesthetic in the stellate ganglion area, which would have excluded the last mechanism. However, considering the absence in the patient of hoarseness or respiratory discomfort, which are symptoms of recurrent laryngeal nerve block or phrenic nerve block, there appears to be a low possibility of nerve block caused by the spreading of large volume of local anesthetic. Taking anatomical aspects into consideration, there is one more mechanism to consider. Sympathetic trunks connect these ganglia to each other, and gray rami communicantes connect the ganglia to the spinal nerves [15]. Performance of the SGB may have an effect on the gray rami communicantes that are connected to the sympathetic ganglion. In the end, the procedure might affect the phrenic nerve to the diaphragm (C3-C5) and the nerve innervating the anterior scalene muscle (C5-C7), which are connected to spinal nerves. We can speculate that there is a possibility of the SGB working on the efferent limb of the hiccup reflex arc by means of the mechanism mentioned above.

We suggest that SGB affects mainly the central hiccup center and the afferent limb of the hiccup reflex arc. We also speculate that SGB results in down regulation of sympathetic activity.

In conclusion, while the precise mechanisms to explain the clinical effectiveness of SGB on hiccups remain to be elucidated, we report therapeutic success with SGB in patients with persistent or intractable hiccups. In addition, further randomized controlled studies will be needed to better understand the mechanisms of action and to prove the efficacy of SGB in the treatment of hiccups prior to widespread clinical application of the technique. As persistent or intractable 
hiccups are relatively rare, studies will need to be multicentered with sufficiently powered samples.

\section{REFERENCES}

1. Steger M, Schneemann M, Fox M. Systemic review: the pathogenesis and pharmacological treatment of hiccups. Aliment Pharmacol Ther 2015; 42: 1037-50.

2. Chang FY, Lu CL. Hiccup: mystery, nature and treatment. J Neurogastroenterol Motil 2012; 18: 123-30.

3. Wilcox SK, Garry A, Johnson MJ. Novel use of amantadine: to treat hiccups. J Pain Symptom Manage 2009; 38: 460-5.

4. Howard RS. Persistent hiccups. BMJ 1992; 305: 1237-8.

5. Kang KN, Park IK, Suh JH, Leem JG, Shin JW. Ultrasound-guided Pulsed Radiofrequency Lesioning of the Phrenic Nerve in a Patient with Intractable Hiccup. Korean J Pain 2010; 23: 198-201.

6. Ge AX, Ryan ME, Giaccone G, Hughes MS, Pavletic SZ. Acupuncture treatment for persistent hiccups in patients with cancer. J Altern Complement Med 2010; 16: 811-6.

7. Sato S, Asakura N, Endo T, Naito H. Cervical epidural block can relieve postoperative intractable hiccups. Anesthesiology 1993; 78: 1184-6.

8. Keane JR. Hiccups due to central nervous system disease: analysis of 71 inpatients. Can J Neurol Sci 2010; 37: 870-2.
9. Becker DE. Nausea, vomiting, and hiccups: a review of mechanisms and treatment. Anesth Prog 2010; 57: 150-6.

10. Elias M. Cervical sympathetic and stellate ganglion blocks. Pain Physician 2000; 3: 294-304.

11. Cha DC, Song JH, Song BH, Lee JH, Shinn HK, Lee HS. A SGB treatment case of a patient's postoperative intractable hiccup: a case report. Anesth Pain Med 2010; 5: 277-9.

12. Uchida K, Tateda T, Hino H. Novel mechanism of action hypothesized for stellate ganglion block related to melatonin. Med Hypotheses 2002; 59: 446-9.

13. Mulvaney SW, McLean B, de Leeuw J. The use of stellate ganglion block in the treatment of panic/anxiety symptoms with combat-related post-traumatic stress disorder; preliminary results of long-term follow-up: a case series. Pain Pract 2010; 10: 359-65.

14. Lipov EG, Joshi JR, Sanders S, Slavin KV. A unifying theory linking the prolonged efficacy of the stellate ganglion block for the treatment of chronic regional pain syndrome (CRPS), hot flashes, and posttraumatic stress disorder (PTSD). Med Hypotheses 2009; 72: 657-61.

15. Glick DB. The autonomic nervous system. In: Miller's Anesthesia. 8th ed. Edited by Miller RD, Cohen NH, Eriksson LI, Fleisher LA, Wiener-Kronish JP, Young WL: Philadelphia, Churchill Livingstone/Elsevier. 2015, pp 346-86. 